ISSN 1392-3196 / e-ISSN 2335-8947

Zemdirbyste-Agriculture, vol. 102, No. 2 (2015), p. 193-200

DOI 10.13080/z-a.2015.102.025

\title{
Cadmium effects on mineral accumulation, antioxidant defence system and gas exchange in cucumber
}

\author{
Sheng SUN ${ }^{1}$, Mei $\mathrm{LI}^{2,3}$, Jinhua ZUO' ${ }^{2}$, Wusheng JIANG ${ }^{2}$, Donghua $\mathrm{LIU}^{2}$ \\ ${ }^{1}$ Tianjin Normal University \\ Tianjin 300387, China \\ ${ }^{2}$ Tianjin Key Laboratory of Animal and Plant Resistance, Tianjin Normal University \\ Tianjin 300387, China \\ E-mail: donghua@mail.zlnet.com.cn \\ ${ }^{3}$ Renhuai No. 4 Middle School \\ Renhuai City 564507, Guizhou Province, China
}

\begin{abstract}
The effects of different concentrations $(10,50,100$ and $500 \mu \mathrm{M})$ of cadmium $(\mathrm{Cd})$ on manganese $(\mathrm{Mn})$ and iron (Fe), lipid peroxidation, activities of antioxidant enzymes and photosynthetic function were investigated in hydroponically grown cucumber (Cucumis sativus L.). Results indicated that cadmium was accumulated primarily in roots. In the roots and shoots, the cadmium content increased with the increasing cadmium concentrations, and induced decrease of manganese significantly $(P<0.005)$. The levels of iron had an increasing trend with increasing cadmium concentration and duration of treatment. Cadmium-induced oxidation stress and lipid peroxidation in C. sativus showed by the increased concentration of MDA (malondialdehyde). Cadmium induced higher SOD (superoxide dismutase) and POD (peroxidase) activities than CAT (catalase) activity, suggesting that SOD and POD provided a better defence mechanism against cadmium-induced oxidative damage in $C$. sativus. The net photosynthetic rate, transpiration rate, stomatal conductance and intercellular $\mathrm{CO}_{2}$ concentration were reduced in the presence of cadmium.
\end{abstract}

Key words: antioxidant enzymes, cadmium, Cucumis sativus, lipid peroxidation, photosynthetic parameters.

\section{Introduction}

Cadmium $(\mathrm{Cd})$ has no known function as a nutrient and is toxic to plants. When taken up by plants, cadmium may cause several physiological, biochemical and structural changes (Benavides et al., 2005). Cadmium has been shown to interfere with the uptake, transport and use of several elements (Álvarez-Fernández, 2014; Sperotto et al., 2014). Cadmium has been shown to be one of the most effective inhibitors of photosynthetic activity. It can enter chloroplasts and disturb chloroplast function by inhibiting the enzymatic activities of chlorophyll biosynthesis and chloroplast structure (Ying et al., 2010). Stomatal opening, a transpiration, photosynthesis and antioxidant metabolism have been reported to be affected by cadmium (Feng et al., 2010; Shi et al., 2010). Cadmium can produce disturbances in plant antioxidant defences giving rise to oxidative stress. Cadmiuminduced production of the ROS (reactive oxygen species), $\mathrm{H}_{2} \mathrm{O}_{2}$ and $\mathrm{O}_{2}^{-}$, can be attributed to the phytotoxic effect of cadmium, but lower levels of ROS can function as signal molecules in the induction of defence genes against cadmium toxicity (Li, Huang, 2014).

Cucumber (Cucumis sativus L.) is an economically important and widely distributed crop. It is reported that fresh fruit of cucumber per $100 \mathrm{~g}$ contains $94-97 \mathrm{~g}$ water, 1.6-4.1 g carbohydrates, 0.4-1.2 g protein, 4-25 mg vitamin $\mathrm{C}$, and it also contains calcium, iron, phosphorus and other minerals (Lin et al., 2012). C. sativus is sensitive to cadmium. Under cadmium stress, its growth was inhibited, reducing the yield and quality of cucumber. C. sativus absorbs cadmium from soils and eventually translocates it to edible parts. Therefore, consumption, either directly or indirectly, of edible parts with high levels of cadmium can be a food safety concern. For the present investigation, we examined the cadmium toxic effects on other mineral accumulation, antioxidant enzymes, malondialdehyde content, leaf gas exchange and photosynthetic parameters in $C$. sativus subjected to various levels of cadmium. It is very important to elucidate cadmium toxicity to $C$. sativus and the possible mechanism of action.

\section{Materials and methods}

Plant cultivation. The seeds of cucumber (Cucumis sativus L., 'Jinyou' No. 35) used in the present investigation were supplied by Tianjin Vegetable Research Institute, China. After soaking for $12 \mathrm{~h}$, they were allowed to germinate and grew in vermiculite in seven containers with $1 / 2$ nutrient solution in a climate chamber with a day/night period of $14 / 10 \mathrm{~h}$, a day/night temperature and humidity regime of $25 / 20^{\circ} \mathrm{C}$ and $55 / 75 \%$ relative humidity, respectively. After the second leaf of $C$. sativus 
seedlings just came out, seedlings were uniformly selected and treated with different concentrations of cadmium -0 , $10,50,100$ and $500 \mu \mathrm{M}$ for $5,10,15$ and 20 days. The solution consisted of $0.75 \mathrm{mmol} \mathrm{L}^{-1} \mathrm{~K}_{2} \mathrm{SO}_{4}, 0.65 \mathrm{mmol} \mathrm{L}^{-1}$ $\mathrm{MgSO}_{4} \times 7 \mathrm{H}_{2} \mathrm{O}, 0.01 \mathrm{mmol} \mathrm{L}^{-1} \mathrm{KCl}, 0.25 \mathrm{mmol} \mathrm{L}^{-1}$ $\mathrm{KH}_{2} \mathrm{PO}_{4}, 2 \mathrm{mmol} \mathrm{L}-1 \mathrm{Ca}\left(\mathrm{NO}_{3}\right)_{2} \times 4 \mathrm{H}_{2} \mathrm{O}, 100 \mu \mathrm{mol} \mathrm{L}^{-1}$ FeEDTA, $10 \mu \mathrm{mol} \mathrm{L}^{-1} \mathrm{H}_{3} \mathrm{BO}_{3}, 1 \mu \mathrm{mol} \mathrm{L}^{-1} \mathrm{MnSO}_{4} \times \mathrm{H}_{2} \mathrm{O}$, $0.1 \mu \mathrm{mol} \mathrm{L}^{-1} \mathrm{CuSO}_{4} \times 5 \mathrm{H}_{2} \mathrm{O}, 0.05 \mu \mathrm{mol} \mathrm{L}^{-1}\left(\mathrm{NH}_{4}\right)_{6} \mathrm{Mo}_{7} \mathrm{O}_{4}$ $\times 4 \mathrm{H}_{2} \mathrm{O}$ and $1 \mu \mathrm{mol} \mathrm{L}^{-1} \mathrm{ZnSO}$ at a pH of $5.5^{4}$ (Zhang et al., 2009). The nutrient solutions were continuously aerated and changed regularly every 5 days until the seedlings were harvested. Cadmium $(\mathrm{Cd})$ was provided as cadmium chloride $\left(\mathrm{CdCl}_{2} \times 2.5 \mathrm{H}_{2} \mathrm{O}\right)$. Cadmium stock solution was prepared in deionized water. The test solutions were changed every 5 days. Meanwhile the $1 / 2$ strength Hoagland solution without cadmium was used for the control plants. Macroscopic observations and all determinations were conducted at the end of each time interval ( 5 days). The seedlings were used to determine contents of $\mathrm{Cd}$ and other minerals, antioxidant enzyme activities, concentration of MDA (malondialdehyde) and photosynthetic parameters in the present investigation.

Determination of cadmium and other minerals. Twenty plantlets from each treatment were harvested based on uniformity of size and colour (removing the greatest and the smallest plantlets and then selected randomly) after 20 days of incubation. The seedlings were removed from solution and washed thoroughly with running tap water for $30 \mathrm{~min}$ and then with deionized water to remove traces of nutrients and cadmium ions from seedling surfaces. Concentrations of cadmium $(\mathrm{Cd})$, manganese $(\mathrm{Mn})$ and iron $(\mathrm{Fe})$ were analyzed using inductively coupled plasma atomic emission spectrometry (ICP-AES) (Leeman Labs Inc., USA).

Determination of antioxidant enzyme activities and MDA concentration. The fresh roots or leaves from each treatment were homogenized in a pestle and mortar with $0.05 \mathrm{M}$ sodium phosphate buffer $(\mathrm{pH} 7.8)$ at the end of each time interval ( 5 days). The homogenate was centrifuged at $10000 \times \mathrm{g}$ for $20 \mathrm{~min}$, and the supernatant was used for analyzing superoxide dismutase (SOD), peroxidase (POD) and catalase (CAT). The above steps were carried out at $4^{\circ} \mathrm{C}$. The methods are described in more detail ( $\mathrm{Li}$ et al., 2013). The fresh leaf $(1 \mathrm{~g})$ in each treatment was homogenized in $10 \mathrm{~mL}$ of $10 \%$ TCA (trichloroacetic acid) with a pestle and mortar. The homogenates were centrifuged at $4000 \times \mathrm{g}$ for 20 min. To $2 \mathrm{~mL}$ aliquot of the supernatant, $2 \mathrm{~mL}$ of $0.6 \%$ thiobarbituric acid in 10\% TCA was added. The mixture was heated at $100^{\circ} \mathrm{C}$ for 15 min and then quickly cooled in an ice bath. After centrifugation at $10000 \times \mathrm{g}$ for 10 min, the absorbance of the supernatant was recorded at 532 and $450 \mathrm{~nm}$. Lipid peroxidation was expressed as MDA content in $1 \mathrm{M} \mathrm{kg}^{-1}$ fresh weight.

Leaf gas exchange and photosynthetic parameter measurements. Leaf gas exchange and photosynthetic parameters: net photosynthetic rate $\left(\mathrm{P}_{\mathrm{n}}\right)$, transpiration rate $\left(\mathrm{T}_{\mathrm{r}}\right)$, stomatal conductance $\left(\mathrm{G}_{\mathrm{s}}\right)$ and intercellular $\mathrm{CO}_{2}$ concentration $\left(\mathrm{C}_{\mathrm{i}}\right)$, were measured in the first fully expanded leaf from the top shoot at the end of each time interval (5 days), using handheld photosynthesis system CI-340 (CID Inc., USA). The measurements were performed between 10:00 and 11:00 a.m. under the greenhouse conditions described above. Data were given and recorded by the machine.

Statistical analysis. In treatment groups, seedlings were examined for macroscopic observations and all determinations at the end of each time interval (5days). Each treatment was run in triplicate for statistical validity. Data from this investigation were analyzed with standard statistical software Sigma Plot 8.0 using means \pm standard error. For equality of averages the $t$-test was applied. Results were considered statistically significant at $p<0.05$.

\section{Results}

Effects of cadmium on seedlings. The seedling growth of $C$. sativus varied with the cadmium concentration and duration of treatment (Fig. 1). During the 10 days, $10 \mu \mathrm{M} \mathrm{Cd}$ exposure had no toxic effect on root length. After 15 days, the root growth was inhibited significantly $(P<0.05)$. In the other three treatment groups the root length was inhibited significantly $(P<0.05)$ during the duration of the experiment when compared to control except for the group treated with $50 \mu \mathrm{M} \mathrm{Cd}$ for 5 days (Fig. $1 \mathrm{~A})$. At lower concentrations of $\mathrm{Cd}$ (10 and $50 \mu \mathrm{M})$, the roots became yellow, and at $500 \mu \mathrm{M}$ $\mathrm{Cd}$, most seedling roots appeared black. The seedlings treated with 10 and $50 \mu \mathrm{M} \mathrm{Cd}$ for 5 days showed the same growth trend as the control. At the 50 to $500 \mu \mathrm{M} \mathrm{Cd}$ dose, the seedling growth was inhibited significantly $(P$ $<0.05)$ during the entire experiment except for the group exposed to $50 \mu \mathrm{M} \mathrm{Cd}$ for 5 days. At 100 and $500 \mu \mathrm{M} \mathrm{Cd}$ concentrations, the seedling growth almost completely stopped during the whole course of treatment (Fig. 1 B).
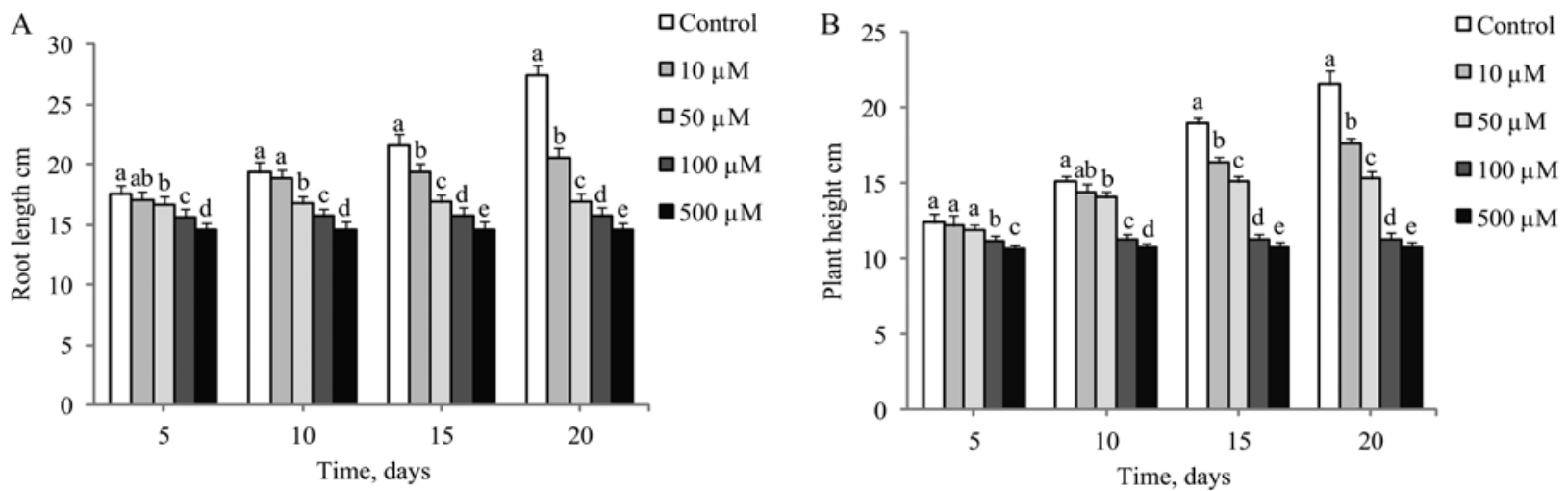

Note. Vertical bars denote standard error, $\mathrm{n}=10$; values with different letters differ significantly from each other $(p<0.05, t$-test).

Figure 1. Effects of different concentrations of cadmium (Cd) on Cucumis sativus seedlings stressed for 5, 10, 15 and 20 days 
Leaves of control plants showed green in colour during the whole experiment. In contrast to control leaves, the serious chlorotic leaves with slight green around veins were exhibited after exposure to cadmium. In the group of plants treated with $500 \mu \mathrm{M} \mathrm{Cd}$, the leaves showed chlorotic symptoms like the plants with irondeficiency (Fig. 2).
Cadmium accumulation and its effect on other minerals. Statistical analysis showed the presence of significant correlations between the concentration of cadmium and microelements (manganese and iron). The accumulation of cadmium in C. sativus roots, stems and shoots varied with cadmium concentration and treatment time. As shown in Table 1, levels of cadmium accumulation



Figure 2. Visible symptoms in leaves of Cucumis sativus exposed to cadmium (Cd) for 20 days (A - control, B $500 \mu \mathrm{M} \mathrm{Cd})$

by roots, stems and shoots increased with increasing cadmium concentration and prolonged treatment time. The accumulation of cadmium ions primarily was in roots, and small amounts of cadmium were transported to stems and leaves. Levels of cadmium in $C$. sativus treated with cadmium were in the order: roots $>$ stems $>$ leaves. In the presence of cadmium, the contents of manganese in roots, stems and leaves of C.sativus decreased with increasing cadmium concentration. Manganese accumulation was inhibited significantly $(P<0.005)$. The levels of iron in roots, stems and leaves of $C$. sativus had an increasing trend with increasing cadmium concentration and duration of treatment.
Effects of cadmium on the activities of SOD, CAT and POD. Effects of cadmium on SOD activities in roots and leaves of $C$. sativus varied with the different concentrations of cadmium and the duration of treatment (Fig. $3 \mathrm{~A}$ and B). Figure $3 \mathrm{~A}$ showed that SOD activities in roots exposed to cadmium during the whole experiment time were observed to be significant $(P<$ $0.05)$ in comparison with control. The activity of SOD in leaves exposed to cadmium increased significantly $(P<0.05)$ during the whole treatment when compared with control (Fig. 3 B). Both controls and cadmium treatments showed higher SOD activity in leaves than in roots. The activities of POD in $C$. sativus are presented

Table 1. Cadmium (Cd), manganese ( $\mathrm{Mn})$ and iron $(\mathrm{Fe})$ accumulation by Cucumis sativus root, stem and leaf

\begin{tabular}{|c|c|c|c|c|c|c|c|c|c|c|}
\hline \multirow{3}{*}{$\begin{array}{l}\text { Time, } \\
\text { days }\end{array}$} & \multirow{3}{*}{$\begin{array}{c}\text { Treatment } \\
\mu \mathrm{M}\end{array}$} & \multicolumn{3}{|c|}{ Root } & \multicolumn{3}{|c|}{ Stem } & \multicolumn{3}{|c|}{ Leaf } \\
\hline & & \multicolumn{9}{|c|}{$\mu \mathrm{g} \mathrm{g}^{-1}$ dry weight \pm standard error } \\
\hline & & $\mathrm{Cd}$ & $\mathrm{Mn}$ & $\mathrm{Fe}$ & $\mathrm{Cd}$ & $\mathrm{Mn}$ & $\mathrm{Fe}$ & $\mathrm{Cd}$ & $\mathrm{Mn}$ & $\mathrm{Fe}$ \\
\hline 1 & 2 & 3 & 4 & 5 & 6 & 7 & 8 & 9 & 10 & 11 \\
\hline \multirow{5}{*}{5} & Control & $\begin{array}{l}0.00 \pm \\
0.00 \mathrm{a}\end{array}$ & $\begin{array}{c}83.16 \pm \\
0.28 \mathrm{a}\end{array}$ & $\begin{array}{l}620 \pm \\
0.36 \mathrm{a}\end{array}$ & $\begin{array}{l}0.00 \pm \\
0.00 \mathrm{a}\end{array}$ & $\begin{array}{c}11.26 \pm \\
0.08 \mathrm{a}\end{array}$ & $\begin{array}{l}5.20 \pm \\
0.06 \mathrm{a}\end{array}$ & $\begin{array}{l}0.00 \pm \\
0.00 \mathrm{a}\end{array}$ & $\begin{array}{l}146 \pm \\
0.26 \mathrm{a}\end{array}$ & $\begin{array}{c}49.53 \pm \\
0.16 \mathrm{a}\end{array}$ \\
\hline & 10 & $\begin{array}{l}3178 \pm \\
16.64 \mathrm{~b}\end{array}$ & $\begin{array}{c}63.18 \pm \\
0.48 \mathrm{~b}\end{array}$ & $\begin{array}{l}1133 \pm \\
3.93 \mathrm{~b}\end{array}$ & $\begin{array}{l}285 \pm \\
1.71 b\end{array}$ & $\begin{array}{l}9.69 \pm \\
0.09 \mathrm{~b}\end{array}$ & $\begin{array}{l}9.90 \pm \\
0.38 \mathrm{~b}\end{array}$ & $\begin{array}{c}82.45 \pm \\
2.06 \mathrm{~b}\end{array}$ & $\begin{array}{c}78.51 \pm \\
0.23 \mathrm{~b}\end{array}$ & $\begin{array}{c}58.27 \pm \\
0.36 \mathrm{~b}\end{array}$ \\
\hline & 50 & $\begin{array}{l}5270 \pm \\
13.87 \mathrm{c}\end{array}$ & $\begin{array}{c}56.84 \pm \\
0.49 c\end{array}$ & $\begin{array}{l}1264 \pm \\
10.19 \mathrm{c}\end{array}$ & $\begin{array}{l}721 \pm \\
1.24 \mathrm{c}\end{array}$ & $\begin{array}{l}7.78 \pm \\
0.03 \mathrm{c}\end{array}$ & $\begin{array}{c}17.69 \pm \\
0.09 \mathrm{c}\end{array}$ & $\begin{array}{l}151 \pm \\
0.75 \mathrm{c}\end{array}$ & $\begin{array}{c}73.47 \pm \\
0.26 \mathrm{c}\end{array}$ & $\begin{array}{c}92.51 \pm \\
1.40 \mathrm{c}\end{array}$ \\
\hline & 100 & $\begin{array}{c}8592 \pm \\
5.26 \mathrm{~d}\end{array}$ & $\begin{array}{c}37.57 \pm \\
0.23 \mathrm{~d}\end{array}$ & $\begin{array}{l}1624 \pm \\
3.51 \mathrm{~d}\end{array}$ & $\begin{array}{l}1185 \pm \\
2.10 \mathrm{~d}\end{array}$ & $\begin{array}{l}7.35 \pm \\
0.05 \mathrm{~d}\end{array}$ & $\begin{array}{c}23.31 \pm \\
0.58 \mathrm{~d}\end{array}$ & $\begin{array}{c}161 \pm \\
0.79 \mathrm{~d}\end{array}$ & $\begin{array}{c}70.81 \pm \\
0.12 \mathrm{~d}\end{array}$ & $\begin{array}{c}118 \pm \\
0.37 \mathrm{~d}\end{array}$ \\
\hline & 500 & $\begin{array}{l}12861 \pm \\
112.05 \mathrm{e} \\
\end{array}$ & $\begin{array}{c}31.71 \pm \\
0.21 \mathrm{e} \\
\end{array}$ & $\begin{array}{c}1745 \pm \\
5.31 \mathrm{e} \\
\end{array}$ & $\begin{array}{c}1833 \pm \\
1.49 \mathrm{e} \\
\end{array}$ & $\begin{array}{l}6.38 \pm \\
0.10 \mathrm{e} \\
\end{array}$ & $\begin{array}{c}31.08 \pm \\
0.38 \mathrm{e} \\
\end{array}$ & $\begin{array}{l}307 \pm \\
0.94 \mathrm{e} \\
\end{array}$ & $\begin{array}{c}67.23 \pm \\
0.38 \mathrm{e} \\
\end{array}$ & $\begin{array}{l}141 \pm \\
0.04 \mathrm{e}\end{array}$ \\
\hline \multirow{5}{*}{10} & Control & $\begin{array}{l}0.00 \pm \\
0.00 \mathrm{a}\end{array}$ & $\begin{array}{c}85.36 \pm \\
0.29 \mathrm{a}\end{array}$ & $\begin{array}{l}1412 \pm \\
8.41 \mathrm{a}\end{array}$ & $\begin{array}{l}0.00 \pm \\
0.00 \mathrm{a}\end{array}$ & $\begin{array}{c}26.42 \pm \\
0.24 \mathrm{a}\end{array}$ & $\begin{array}{c}12.41 \pm \\
0.65 \mathrm{a}\end{array}$ & $\begin{array}{l}0.00 \pm \\
0.00 \mathrm{a}\end{array}$ & $\begin{array}{l}154 \pm \\
0.03 \mathrm{a}\end{array}$ & $\begin{array}{c}98.47 \pm \\
0.04 \mathrm{a}\end{array}$ \\
\hline & 10 & $\begin{array}{l}4445 \pm \\
9.25 \mathrm{~b}\end{array}$ & $\begin{array}{c}71.87 \pm \\
0.15 \mathrm{~b}\end{array}$ & $\begin{array}{l}1619 \pm \\
3.85 \mathrm{~b}\end{array}$ & $\begin{array}{l}363 \pm \\
3.02 \mathrm{~b}\end{array}$ & $\begin{array}{c}21.52 \pm \\
0.04 \mathrm{~b}\end{array}$ & $\begin{array}{c}17.62 \pm \\
0.75 \mathrm{~b}\end{array}$ & $\begin{array}{l}163 \pm \\
0.46 \mathrm{~b}\end{array}$ & $\begin{array}{c}76.31 \pm \\
0.25 \mathrm{~b}\end{array}$ & $\begin{array}{l}101 \pm \\
0.14 b\end{array}$ \\
\hline & 50 & $\begin{array}{l}6295 \pm \\
14.24 \mathrm{c}\end{array}$ & $\begin{array}{c}61.94 \pm \\
0.23 \mathrm{c}\end{array}$ & $\begin{array}{l}1842 \pm \\
12.09 \mathrm{c}\end{array}$ & $\begin{array}{l}919 \pm \\
1.83 \mathrm{c}\end{array}$ & $\begin{array}{c}19.12 \pm \\
0.15 \mathrm{c}\end{array}$ & $\begin{array}{c}21.57 \pm \\
0.78 \mathrm{c}\end{array}$ & $\begin{array}{l}243 \pm \\
0.63 \mathrm{c}\end{array}$ & $\begin{array}{c}70.62 \pm \\
0.33 \mathrm{c}\end{array}$ & $\begin{array}{l}105 \pm \\
0.22 \mathrm{c}\end{array}$ \\
\hline & 100 & $\begin{array}{c}10075 \pm \\
15.47 \mathrm{~d}\end{array}$ & $\begin{array}{c}48.69 \pm \\
0.09 \mathrm{~d}\end{array}$ & $\begin{array}{c}2014 \pm \\
7.81 \mathrm{~d}\end{array}$ & $\begin{array}{c}1224 \pm \\
0.18 \mathrm{~d}\end{array}$ & $\begin{array}{c}16.47 \pm \\
0.17 \mathrm{~d}\end{array}$ & $\begin{array}{c}24.97 \pm \\
0.57 \mathrm{~d}\end{array}$ & $\begin{array}{l}262 \pm \\
1.49 \mathrm{~d}\end{array}$ & $\begin{array}{c}63.97 \pm \\
0.10 \mathrm{~d}\end{array}$ & $\begin{array}{c}122 \pm \\
0.29 \mathrm{~d}\end{array}$ \\
\hline & 500 & $\begin{array}{l}13936 \pm \\
22.66 \mathrm{e}\end{array}$ & $\begin{array}{c}35.03 \pm \\
0.14 \mathrm{e}\end{array}$ & $\begin{array}{c}2329 \pm \\
3.98 \mathrm{e}\end{array}$ & $\begin{array}{c}2218 \pm \\
1.91 \mathrm{e}\end{array}$ & $\begin{array}{c}14.40 \pm \\
0.10 \mathrm{e}\end{array}$ & $\begin{array}{c}32.75 \pm \\
0.48 \mathrm{e}\end{array}$ & $\begin{array}{l}362 \pm \\
0.81 \mathrm{e}\end{array}$ & $\begin{array}{c}48.58 \pm \\
0.04 \mathrm{e}\end{array}$ & $\begin{array}{l}154 \pm \\
0.54 \mathrm{e}\end{array}$ \\
\hline
\end{tabular}


Table 2 continued

\begin{tabular}{|c|c|c|c|c|c|c|c|c|c|c|}
\hline 1 & 2 & 3 & 4 & 5 & 6 & 7 & 8 & 9 & 10 & 11 \\
\hline \multirow{5}{*}{15} & Control & $\begin{array}{l}0.00 \pm \\
0.00 \mathrm{a}\end{array}$ & $\begin{array}{c}125 \pm \\
0.21 \mathrm{a}\end{array}$ & $\begin{array}{c}1821 \pm \\
10.07 \mathrm{a}\end{array}$ & $\begin{array}{l}0.00 \pm \\
0.00 \mathrm{a}\end{array}$ & $\begin{array}{c}30.56 \pm \\
0.12 \mathrm{a}\end{array}$ & $\begin{array}{c}15.99 \pm \\
0.33 \mathrm{a}\end{array}$ & $\begin{array}{l}0.00 \pm \\
0.00 \mathrm{a}\end{array}$ & $\begin{array}{l}163 \pm \\
2.29 \mathrm{a}\end{array}$ & $\begin{array}{l}100 \pm \\
0.38 \mathrm{a}\end{array}$ \\
\hline & 10 & $\begin{array}{c}5147 \pm \\
4.65 \mathrm{~b}\end{array}$ & $\begin{array}{c}98.59 \pm \\
0.35 \mathrm{~b}\end{array}$ & $\begin{array}{l}2151 \pm \\
13.57 \mathrm{~b}\end{array}$ & $\begin{array}{l}501 \pm \\
4.34 \mathrm{~b}\end{array}$ & $\begin{array}{c}25.48 \pm \\
0.10 \mathrm{~b}\end{array}$ & $\begin{array}{l}18.17 \pm \\
0.419 b\end{array}$ & $\begin{array}{l}217 \pm \\
1.19 b\end{array}$ & $\begin{array}{c}70.34 \pm \\
0.02 \mathrm{~b}\end{array}$ & $\begin{array}{l}105 \pm \\
1.41 \mathrm{~b}\end{array}$ \\
\hline & 50 & $\begin{array}{l}7772 \pm \\
23.01 \mathrm{c}\end{array}$ & $\begin{array}{c}64.60 \pm \\
0.32 \mathrm{c}\end{array}$ & $\begin{array}{l}2200 \pm \\
10.25 \mathrm{c}\end{array}$ & $\begin{array}{c}1191 \pm \\
6.24 \mathrm{c}\end{array}$ & $\begin{array}{c}21.44 \pm \\
0.04 \mathrm{c}\end{array}$ & $\begin{array}{c}23.51 \pm \\
0.16 \mathrm{c}\end{array}$ & $\begin{array}{l}293 \pm \\
0.44 \mathrm{c}\end{array}$ & $\begin{array}{c}60.07 \pm \\
0.02 \mathrm{c}\end{array}$ & $\begin{array}{l}112 \pm \\
0.25 \mathrm{c}\end{array}$ \\
\hline & 100 & $\begin{array}{l}11332 \pm \\
18.93 \mathrm{~d}\end{array}$ & $\begin{array}{c}53.02 \pm \\
0.20 \mathrm{~d}\end{array}$ & $\begin{array}{l}2336 \pm \\
10.37 \mathrm{~d}\end{array}$ & $\begin{array}{l}1377 \pm \\
11.81 \mathrm{~d}\end{array}$ & $\begin{array}{c}19.20 \pm \\
0.06 \mathrm{~d}\end{array}$ & $\begin{array}{c}26.86 \pm \\
0.16 \mathrm{~d}\end{array}$ & $\begin{array}{l}333 \pm \\
1.30 \mathrm{~d}\end{array}$ & $\begin{array}{c}57.99 \pm \\
0.16 \mathrm{c}\end{array}$ & $\begin{array}{l}140 \pm \\
1.53 \mathrm{~d}\end{array}$ \\
\hline & 500 & $\begin{array}{c}18829 \pm \\
33.27 \mathrm{e}\end{array}$ & $\begin{array}{c}37.44 \pm \\
0.35 \mathrm{e} \\
\end{array}$ & $\begin{array}{c}3080 \pm \\
7.06 \mathrm{e} \\
\end{array}$ & $\begin{array}{l}2697 \pm \\
20.57 \mathrm{e}\end{array}$ & $\begin{array}{c}18.66 \pm \\
0.05 \mathrm{e}\end{array}$ & $\begin{array}{c}40.15 \pm \\
0.27 \mathrm{e} \\
\end{array}$ & $\begin{array}{l}385 \pm \\
0.94 \mathrm{e}\end{array}$ & $\begin{array}{c}45.16 \pm \\
0.24 \mathrm{~d} \\
\end{array}$ & $\begin{array}{l}159 \pm \\
1.54 \mathrm{e}\end{array}$ \\
\hline \multirow{5}{*}{20} & Control & $\begin{array}{l}0.00 \pm \\
0.00 \mathrm{a}\end{array}$ & $\begin{array}{l}208 \pm \\
1.19 \mathrm{a}\end{array}$ & $\begin{array}{c}2190 \pm \\
1.57 \mathrm{a}\end{array}$ & $\begin{array}{l}0.00 \pm \\
0.00 \mathrm{a}\end{array}$ & $\begin{array}{c}33.17 \pm \\
0.07 \mathrm{a}\end{array}$ & $\begin{array}{c}19.34 \pm \\
0.19 \mathrm{a}\end{array}$ & $\begin{array}{l}0.00 \pm \\
0.00 \mathrm{a}\end{array}$ & $\begin{array}{l}169 \pm \\
0.45 \mathrm{a}\end{array}$ & $\begin{array}{l}122 \pm \\
1.23 \mathrm{a}\end{array}$ \\
\hline & 10 & $\begin{array}{c}7049 \pm \\
10.50 \mathrm{~b}\end{array}$ & $\begin{array}{c}104 \pm \\
0.39 \mathrm{~b}\end{array}$ & $\begin{array}{l}2581 \pm \\
21.88 \mathrm{~b}\end{array}$ & $\begin{array}{l}588 \pm \\
7.46 \mathrm{~b}\end{array}$ & $\begin{array}{c}25.74 \pm \\
0.10 \mathrm{~b}\end{array}$ & $\begin{array}{c}22.38 \pm \\
0.23 \mathrm{~b}\end{array}$ & $\begin{array}{l}241 \pm \\
1.33 \mathrm{~b}\end{array}$ & $\begin{array}{c}54.59 \pm \\
0.07 \mathrm{~b}\end{array}$ & $\begin{array}{l}128 \pm \\
0.48 b\end{array}$ \\
\hline & 50 & $\begin{array}{l}9596 \pm \\
16.02 \mathrm{c}\end{array}$ & $\begin{array}{c}70.72 \pm \\
0.12 \mathrm{c}\end{array}$ & $\begin{array}{c}2817 \pm \\
4.60 \mathrm{c}\end{array}$ & $\begin{array}{c}1348 \pm \\
5.69 \mathrm{c}\end{array}$ & $\begin{array}{c}22.20 \pm \\
0.20 \mathrm{c}\end{array}$ & $\begin{array}{c}24.59 \pm \\
0.23 \mathrm{c}\end{array}$ & $\begin{array}{l}329 \pm \\
0.46 \mathrm{c}\end{array}$ & $\begin{array}{c}43.96 \pm \\
0.23 \mathrm{c}\end{array}$ & $\begin{array}{l}137 \pm \\
0.39 c\end{array}$ \\
\hline & 100 & $\begin{array}{l}12471 \pm \\
41.52 \mathrm{~d}\end{array}$ & $\begin{array}{c}65.78 \pm \\
0.42 \mathrm{~d}\end{array}$ & $\begin{array}{l}3537 \pm \\
42.98 \mathrm{~d}\end{array}$ & $\begin{array}{l}1651 \pm \\
2.32 \mathrm{~d}\end{array}$ & $\begin{array}{c}19.36 \pm \\
0.11 \mathrm{~d}\end{array}$ & $\begin{array}{c}37.73 \pm \\
0.76 \mathrm{~d}\end{array}$ & $\begin{array}{l}387 \pm \\
1.15 \mathrm{~d}\end{array}$ & $\begin{array}{c}21.54 \pm \\
0.20 \mathrm{~d}\end{array}$ & $\begin{array}{l}142 \pm \\
0.56 \mathrm{~d}\end{array}$ \\
\hline & 500 & $\begin{array}{c}25741 \pm \\
83.77 \mathrm{e}\end{array}$ & $\begin{array}{c}39.57 \pm \\
0.27 \mathrm{e}\end{array}$ & $\begin{array}{l}5050 \pm \\
12.29 \mathrm{e}\end{array}$ & $\begin{array}{l}3603 \pm \\
10.75 \mathrm{e}\end{array}$ & $\begin{array}{c}18.98 \pm \\
0.07 \mathrm{~d}\end{array}$ & $\begin{array}{c}54.34 \pm \\
0.17 \mathrm{e}\end{array}$ & $\begin{array}{l}409 \pm \\
1.32 \mathrm{e}\end{array}$ & $\begin{array}{c}19.25 \pm \\
0.07 \mathrm{e}\end{array}$ & $\begin{array}{l}170 \pm \\
0.75 \mathrm{e}\end{array}$ \\
\hline
\end{tabular}

Note. Values followed by same letters are not significantly different $(P<0.005)$; means \pm standard error, $\mathrm{n}=10$.
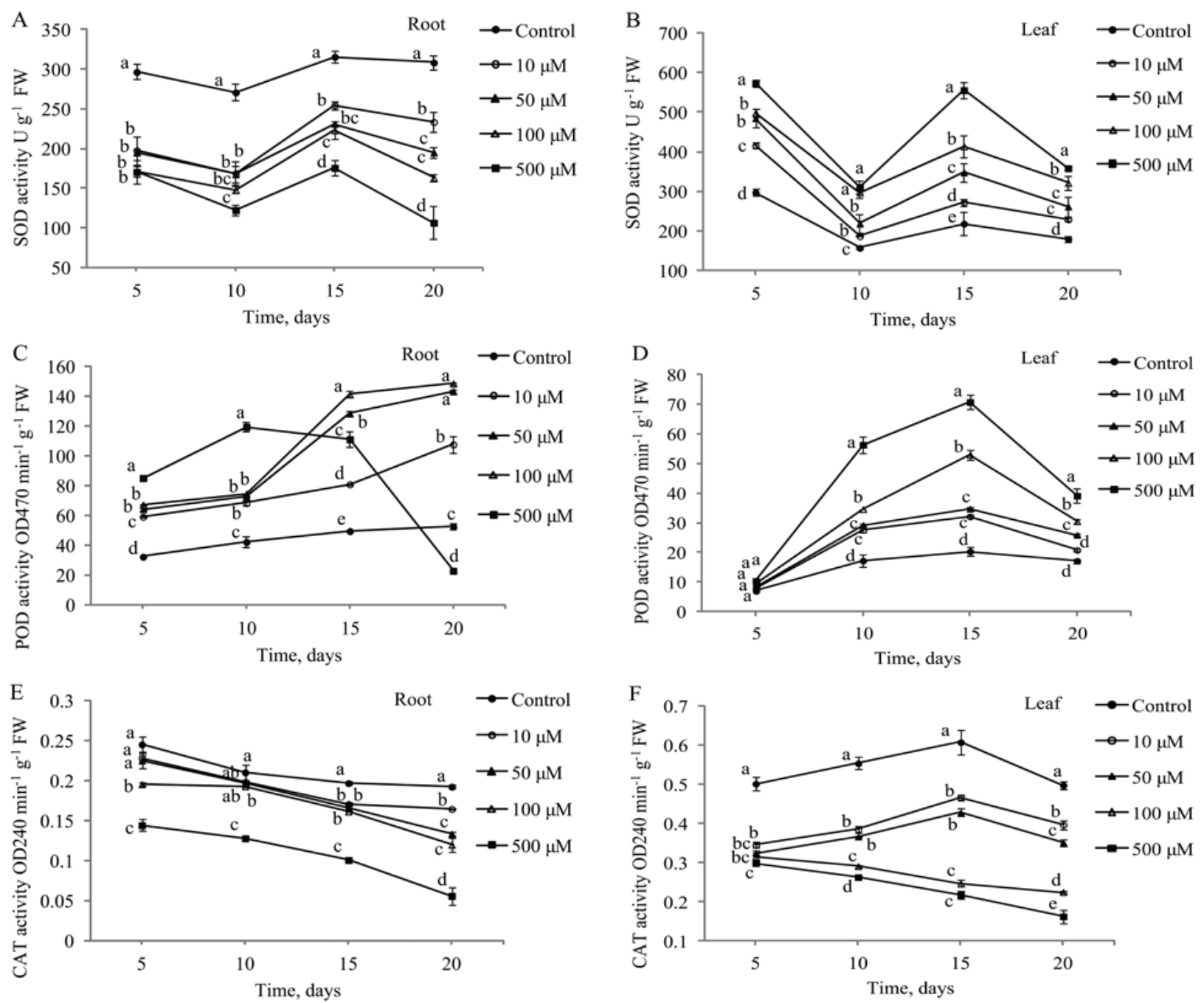

Explanations under Figure 1

Figure 3. Effects of different cadmium (Cd) concentrations on the activities of SOD (superoxide dismutase), POD (peroxidase) and CAT (catalase) in roots and leaves of Cucumis sativus 
in Figure $3 \mathrm{C}$ and $\mathrm{D}$ when the seedlings were exposed to different concentrations of cadmium. In roots, cadmium induced significantly high POD activity during the whole experiment time except for the group exposed to $500 \mu \mathrm{M} \mathrm{Cd}$ at $20^{\text {th }}$ day (Fig. $3 \mathrm{C}$ ). In leaves, the presence of cadmium in the solution increased markedly POD activity $(P<0.05)$ at the $10^{\text {th }}, 15^{\text {th }}$ and $20^{\text {th }}$ days in comparison with control (Fig. 3 D). Information on CAT activity was given in Figure $3 \mathrm{E}$ and $\mathrm{F}$. In roots, the CAT activities decreased significantly $(P<0.05)$ with increasing cadmium concentration and treatment time, except for the groups treated with 10 and $50 \mu \mathrm{M} \mathrm{Cd}$ during 10 days treatment when compared to control (Fig. 3 E). At high concentration of $\mathrm{Cd}(500 \mu \mathrm{M})$, the CAT activity was severely inhibited during the whole experiment in comparison with control and the other treatment groups. The CAT activity was found to be inhibited significantly $(P<0.05)$ during 20 days treatment and to be the lowest in the leaves at $500 \mu \mathrm{M}$ Cd when compared with control and other treatment groups (Fig. $3 \mathrm{~F}$ ).

Effects of cadmium on lipid peroxidation. The effects of cadmium on MDA concentration are presented in Figure $4 \mathrm{~A}$ and $\mathrm{B}$. The MDA contents in roots exposed

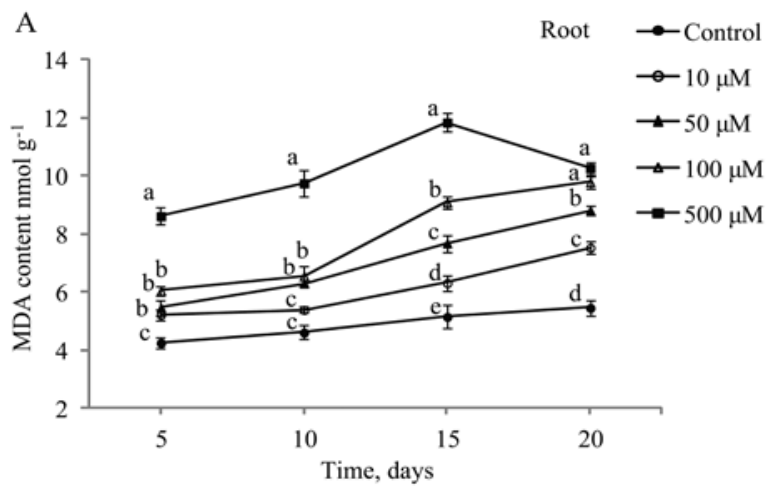

Explanations under Figure 1

Figure 4. Effects of different cadmium (Cd) concentrations on the contents of MDA (malondialdehyde) in roots and leaves of Cucumis sativus

Table 2. Effects of different cadmium concentrations on net photosynthetic rate $\left(\mathrm{P}_{\mathrm{n}}\right)$, transpiration rate $\left(\mathrm{T}_{\mathrm{r}}\right)$, intercellular $\mathrm{CO}_{2}$ concentration $\left(\mathrm{C}_{\mathrm{i}}\right)$ and stomatal conductance $\left(\mathrm{G}_{\mathrm{s}}\right)$ in Cucumis sativus

\begin{tabular}{|c|c|c|c|c|c|}
\hline \multirow{2}{*}{$\begin{array}{l}\text { Time, } \\
\text { days }\end{array}$} & \multirow{2}{*}{$\begin{array}{c}\text { Treatment } \\
\mu \mathrm{M}\end{array}$} & \multicolumn{4}{|c|}{ Photosynthetic gas exchange } \\
\hline & & 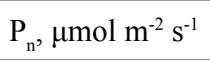 & 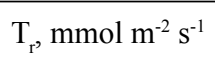 & $\mathrm{C}_{\mathrm{i}}, \mu \mathrm{mol} \mathrm{\textrm {mol } ^ { - 1 }}$ & $\mathrm{G}_{\mathrm{s}}, \mu \mathrm{mol} \mathrm{m} \mathrm{m}^{-2} \mathrm{~s}^{-1}$ \\
\hline \multirow{5}{*}{5} & control & $7.05 \pm 0.26 \mathrm{a}$ & $3.45 \pm 0.25 \mathrm{a}$ & $506 \pm 6.85 \mathrm{a}$ & $114 \pm 5.38 \mathrm{a}$ \\
\hline & $10 \mu \mathrm{M}$ & $6.29 \pm 0.10 \mathrm{ab}$ & $2.77 \pm 0.75 \mathrm{~b}$ & $483 \pm 3.99 \mathrm{ab}$ & $97.57 \pm 3.79 \mathrm{a}$ \\
\hline & $50 \mu \mathrm{M}$ & $5.49 \pm 0.25 b$ & $1.94 \pm 0.06 \mathrm{~b}$ & $445 \pm 6.59 b$ & $73.02 \pm 3.91 \mathrm{~b}$ \\
\hline & $100 \mu \mathrm{M}$ & $3.20 \pm 0.13 \mathrm{c}$ & $1.44 \pm 0.07 \mathrm{c}$ & $408 \pm 2.21 \mathrm{c}$ & $58.30 \pm 2.81 \mathrm{~b}$ \\
\hline & $500 \mu \mathrm{M}$ & $2.41 \pm 0.09 \mathrm{~d}$ & $0.98 \pm 0.05 \mathrm{~d}$ & $387 \pm 7.47 \mathrm{c}$ & $31.60 \pm 1.49 \mathrm{c}$ \\
\hline \multirow{5}{*}{10} & control & $9.47 \pm 0.25 \mathrm{a}$ & $3.51 \pm 0.12 \mathrm{a}$ & $535 \pm 3.46 \mathrm{a}$ & $138 \pm 6.71 \mathrm{a}$ \\
\hline & $10 \mu \mathrm{M}$ & $7.34 \pm 0.24 b$ & $2.69 \pm 0.11 b$ & $496 \pm 4.16 b$ & $105 \pm 2.36 \mathrm{~b}$ \\
\hline & $50 \mu \mathrm{M}$ & $6.57 \pm 0.28 b$ & $1.81 \pm 0.07 \mathrm{c}$ & $458 \pm 3.13 b$ & $83.33 \pm 3.30 \mathrm{c}$ \\
\hline & $100 \mu \mathrm{M}$ & $3.32 \pm 0.12 \mathrm{c}$ & $1.16 \pm 0.06 \mathrm{~d}$ & $414 \pm 5.36 \mathrm{c}$ & $66.78 \pm 2.47 \mathrm{c}$ \\
\hline & $500 \mu \mathrm{M}$ & $1.86 \pm 0.07 \mathrm{~d}$ & $0.86 \pm 0.04 \mathrm{e}$ & $383 \pm 2.15 \mathrm{~d}$ & $29.26 \pm 1.42 \mathrm{~d}$ \\
\hline \multirow{5}{*}{15} & control & $11.12 \pm 0.13 \mathrm{a}$ & $3.57 \pm 0.06 \mathrm{a}$ & $547 \pm 2.15 \mathrm{a}$ & $157 \pm 4.53 \mathrm{a}$ \\
\hline & $10 \mu \mathrm{M}$ & $8.35 \pm 0.12 b$ & $2.63 \pm 0.11 b$ & $513 \pm 3.47 b$ & $114 \pm 5.29 b$ \\
\hline & $50 \mu \mathrm{M}$ & $7.16 \pm 0.08 \mathrm{c}$ & $1.71 \pm 0.08 \mathrm{~b}$ & $464 \pm 5.72 c$ & $89.74 \pm 3.54 \mathrm{c}$ \\
\hline & $100 \mu \mathrm{M}$ & $3.21 \pm 0.12 \mathrm{~d}$ & $1.13 \pm 0.02 \mathrm{c}$ & $415 \pm 9.91 \mathrm{~d}$ & $64.81 \pm 1.97 \mathrm{~d}$ \\
\hline & $500 \mu \mathrm{M}$ & $1.12 \pm 0.08 \mathrm{e}$ & $0.59 \pm 0.03 \mathrm{~d}$ & $373 \pm 6.81 \mathrm{e}$ & $23.46 \pm 1.11 \mathrm{e}$ \\
\hline \multirow{5}{*}{20} & control & $12.66 \pm 0.31 \mathrm{a}$ & $3.71 \pm 0.15 \mathrm{a}$ & $565 \pm 5.41 \mathrm{a}$ & $177 \pm 8.14 \mathrm{a}$ \\
\hline & $10 \mu \mathrm{M}$ & $9.49 \pm 0.22 b$ & $2.50 \pm 0.12 b$ & $515 \pm 3.11 b$ & $118 \pm 5.49 b$ \\
\hline & $50 \mu \mathrm{M}$ & $7.46 \pm 0.12 \mathrm{c}$ & $1.42 \pm 0.06 \mathrm{c}$ & $458 \pm 6.64 c$ & $81.77 \pm 3.40 \mathrm{c}$ \\
\hline & $100 \mu \mathrm{M}$ & $2.57 \pm 0.25 \mathrm{~d}$ & $0.95 \pm 0.03 \mathrm{~d}$ & $4063 \pm 3.00 \mathrm{~d}$ & $54.44 \pm 1.31 \mathrm{~d}$ \\
\hline & $500 \mu \mathrm{M}$ & $0.81 \pm 0.03 \mathrm{e}$ & $0.44 \pm 0.02 \mathrm{e}$ & $340 \pm 16.75 \mathrm{e}$ & $20.13 \pm 0.87 \mathrm{e}$ \\
\hline
\end{tabular}

Note. Values with different letters differ significantly from each other $(p<0.05, t$-test $)$; vertical bars denote standard error, $\mathrm{n}=10$. 
concentration in leaves of $C$. sativus treated with cadmium used in the present investigation during cadmium stress when compared with control (Table 2).

Cadmium had most toxic effects on intercellular $\mathrm{CO}_{2}$ concentration in leaves exposed to $500 \mu \mathrm{M} \mathrm{Cd}$ in comparison with control and the other treatment groups. The stomatal conductance in control leaves was the highest during 20 day treatment. After cadmium treatment, the stomatal conductance in all cadmium treatment groups was inhibited with increasing cadmium concentration except for the group exposed to cadmium for 5 days (Table 2). The stomatal conductance in $500 \mu \mathrm{M}$ $\mathrm{Cd}$ treatment was the lowest during periods of treatment and decreased with prolonged during of treatment.

\section{Discussion}

The results in the present investigation indicate that cadmium inhibits the seedling growth of $C$. sativus significantly $(P<0.05)$ at the concentrations of cadmium from 10 to $500 \mu \mathrm{M}$ during the whole experiment except for the groups exposed to 10 and $50 \mu \mathrm{M}$ Cd for 5 days and $10 \mu \mathrm{M} \mathrm{Cd}$ for 10 days. Chlorosis leaf rolls and stunting are the main and easily visible symptoms of cadmium toxicity in plants (Benavides et al., 2005). We observed that the leaves exposed to cadmium appeared chlorotic with slight green around veins. In the group of plants treated with $500 \mu \mathrm{M} \mathrm{Cd}$, the leaves showed chlorotic symptoms like the plants with iron-deficiency. These phenomena might result from pigment and cell destruction in leaves. Çanakci and Karaboğa (2013) found that the chlorophyll content was reduced in cadmium-treated $C$. sativus plants, indicating that the increase in photosynthetic pigment destruction was a typical consequence of heavy metal toxicity.

Normally, cadmium ions are mainly retained in the roots, and only small amounts are transported to the shoots except for hyperaccumulators (Benavides et al., 2005). C. sativus has the ability to accumulate cadmium primarily in its roots, and transport and concentrate it in its stems and levels in much lesser concentrations. This distribution is due to the mobilization of the protective mechanisms of plants, which inhibits the transport to further tissues and organs. These differences in root and shoot accumulation can possibly be explained by the fact that one of the normal functions of roots is to selectively acquire ions from the soil solution, whereas shoot tissue does not normally play this role (Zou et al., 2008). Poor translocation of cadmium to the shoots could be due to sequestration of most of the cadmium in the vacuoles of the root cells to render it nontoxic, which might be a natural toxicity response of the plant (Tewari et al., 2008). Most recognized standard criteria based on metal concentrations in aboveground tissue of plant material were sampled from its natural habitat. Hyperaccumulators have been known to accumulate cadmium above $0.01 \%$ dry tissue $\left(100 \mu \mathrm{g} \mathrm{g}^{-1}\right)$; whereas the normal range of cadmium concentrations in leaf tissue (dry weight) of some species is 0.05 to $0.2 \mu \mathrm{g} \mathrm{g}^{-1}$ (Bao et al., 2011). C. sativus could not be an efficient phytoextraction plant with considerable ability to accumulate cadmium.

Cadmium can alter the uptake of minerals by plants through its effects on the availability of minerals from the soil, or through a reduction in the population of soil microbes (Benavides et al., 2005). Cadmium toxicity may result from disturbance in plant metabolism as a consequence of disturbance in the accumulation and translocation of mineral nutrients. Availability of manganese to plants decreases in the presence of cadmium in soil (Nazar et al., 2012). The manganesemediated amelioration of cadmium-induced root growth inhibition in maize seedlings was shown to be associated with parallel reductions in cadmium accumulation (Pal'ove-Balang et al., 2006). The results from this investigation showed that manganese accumulation was reduced significantly $(P<0.05)$ in $C$. sativus treated with different cadmium concentrations and progressively decreased with an increase in cadmium concentration. The decrease of manganese concentration in leaves under cadmium stress was the key reason for the restraint of leaf photosynthesis (Nazar et al., 2012). Dong et al. (2006) through regression analysis showed that there was a significantly negative correlation between cadmium and manganese, implying the antagonistic effect of cadmium on manganese absorption and translocation. It was reported that chlorosis might reduce manganese transport (Benavides et al., 2005). In the presence of cadmium, adding manganese to the solution significantly improved the plant growth and reduced the concentrations of cadmium in all organs of the plant (Peng et al., 2008). Iron accumulation in the groups treated with cadmium was greater than in control plants in the present investigation. This is in agreement with the findings of Wang et al. (2007), where iron in maize plants was higher than in control plants after treatment with cadmium.

In plants there are protective enzymatic mechanisms and non-enzymatic mechanisms to scavenge ROS (reactive oxygen species) and alleviate their deleterious effects. ROS can be extremely harmful to organisms at high concentrations. ROS can oxidize proteins, lipids, and nucleic acids, often leading to alterations in cell structure and mutagenesis (Scandalios, 2005). Chakraborty et al. (2014) indicated that cadmium accumulation could induce oxidative stress characterized by $\mathrm{H}_{2} \mathrm{O}_{2} / \mathrm{O}_{2}^{-}$production, lipid peroxidation and protein oxidation. Heavy metals cause oxidative damage to plants either directly or indirectly through the formation of ROS, which cause further severe oxidative damage to different cell organelles and biomolecules (Nagajyoti et al., 2010). To resist oxidative stress, plants can induce a series of detoxification reaction catalyzed by antioxidative enzymes, including SOD, POD and CAT (Zhang et al., 2005). SOD is the cell's first line of defence against ROS as the superoxide radical is a precursor to several other highly reactive species so that control over the steady state of superoxide concentration by SOD constitutes an important protective mechanism (Zhang et al., 2009). The results showed that the SOD activity in leaves and roots exposed to cadmium was noted to be high significantly $(P<0.05)$. High SOD activity has been associated with stress tolerance in plants. This may be attributed to the increased production of superoxide, resulting in the activation of existing enzyme pools or increased expression of genes encoding SOD (Zhang et al., 2009). POD is known to play a significant role in oxidative stress conditions, and it has been shown that POD activity can be used as a potential biomarker for sublethal metal toxicity in examined plant species (Gao et al., 2008). POD is also an important enzyme, able to scavenge $\mathrm{H}_{2} \mathrm{O}_{2}$, which is a major substance degraded by SOD. Our results indicated that cadmium induced POD activity in roots and leaves significantly during the whole 
experiment time except for the group in roots exposed to $500 \mu \mathrm{M} \mathrm{Cd}$ at $20^{\text {th }}$ day and the groups in leaves treated with cadmium at $5^{\text {th }}$ day. Increase in POD activity suggested that cadmium directly caused excessive production of $\mathrm{H}_{2} \mathrm{O}_{2}$ in seedlings and/or increased $\mathrm{H}_{2} \mathrm{O}_{2}$ was due to SOD. Thus, increased POD activity, in turn, scavenged excessive $\mathrm{H}_{2} \mathrm{O}$ and damage was limited. CAT is the most universal oxidoreductase, which scavenges $\mathrm{H}_{2} \mathrm{O}_{2}$ to $\mathrm{O}_{2}$ and $\mathrm{H}_{2} \mathrm{O}$. The CAT activity was found to be inhibited significantly $(P<0.05)$ in leaves and roots in the present investigation. As far as sensitivity to heavy metal stress was concerned, CAT was the highest. So the activity of CAT was inhibited firstly under heavy metal stress conditions, which led to $\mathrm{H}_{2} \mathrm{O}_{2}$ clearing blocked (Lin et al., 2012). In this present investigation, CAT activity decreased at the high concentrations of cadmium, indicating that the CAT to eliminate ROS was limited. Cadmium induced higher SOD and POD activities than CAT activity, suggesting that SOD and POD provided a better defence mechanism against cadmium-induced oxidative damage in $C$. sativus.

MDA, one of the decomposition products of membrane polyunsaturated fatty acids, is regarded as a reliable indicator of oxidative stress (Zou et al., 2009). Evidence here demonstrates that cadmium toxicity enhances lipid peroxidation in plant cells, reflected by increased MDA concentration. The cadmium accumulation in $C$. sativus leads to various physiological and biochemical changes. The high contents of MDA in the investigation suggested that cadmium exposure might lead to more ROS in C. sativus. The elimination of ROS mainly depended on the antioxidative defence enzymes. The results further suggested that cadmium exposure might depress the activities of antioxidative enzymes.

The progressive decrease of photosynthetic rate, transpiration rate, stomatal conductance and intercellular $\mathrm{CO}_{2}$ concentration in association with increasing cadmium concentration was observed in the present study. It is supposed that the closure of stomata reduced the photosynthesis (Turner et al., 2007). Ying et al. (2010) indicated that the decrease of photosynthetic and gas exchange parameters, especially intercellular $\mathrm{CO}_{2}$ concentration, stomatal conductance and transpiration rate, could be ascribed to the abnormality of stomata, like stomatal closure, less stoma density and high stomatal resistance, in high cadmium-exposed Picris divaricata. The parallel changes of photosynthetic rate, stomatal conductance, intercellular $\mathrm{CO}_{2}$ concentration and transpiration rate provide evidence that maintenance of photosynthetic rate mainly was attributed to the stomatal conductance, which later controls the transpiration. A reduction in photosynthesis in C. sativus in cadmiumtreated plants was caused by a series of processes, including stomatal and non-stomatal limitations during cadmium stress. The reduction in the rate of photosynthesis denotes that in addition to depressed growth, the cadmium interferes with the photosynthetic activity by directly interfering in the process of photosynthesis. Ci et al. (2010) found a similar inhibiting effect in leaves of wheat. Inhibition of photosynthesis induced by cadmium may also result in lower chlorophyll content. Further studies are needed to clarify this effect.

\section{Conclusion}

Effects of cadmium (Cd) on cucumber (Cucumis sativus L.) were evaluated in this investigation by accumulation of cadmium and its effect on other minerals, antioxidant enzymes, MDA (malondialdehyde) content and leaf gas exchange and photosynthetic parameters. Cadmium was accumulated primarily in roots and it induced decrease of manganese and increase of iron. Cadmium induced higher SOD (superoxide dismutase) and POD (peroxidase) activities than CAT (catalase) activity, suggesting that SOD and POD provided a better defence mechanism against cadmium-induced oxidative damage in cucumber. Cadmium increased concentration of MDA. The net photosynthetic rate $\left(\mathrm{P}_{\mathrm{n}}\right)$, transpiration rate $\left(\mathrm{T}_{r}\right)$, stomatal conductance $\left(\mathrm{G}_{\mathrm{s}}\right)$ and intercellular $\mathrm{CO}_{2}$ concentration $\left(\mathrm{C}_{\mathrm{i}}\right)$ were reduced in the presence of cadmium. The information available in this work is an important step towards obtaining a better understanding of photosynthetic parameters and antioxidant defence system caused by cadmium.

\section{Acknowledgements}

This project was supported by the National Natural Science Foundation of China. The authors wish to express their appreciation to the reviewers for this paper.

Received 28062014 Accepted 31122014

\section{References}

Álvarez-Fernández A., Díaz-Benito P., Abadía A., LópezMillán A. F., Abadía J. 2014. Metal species involved in long distance metal transport in plants. Frontiers in Plant Science, 5: 105 http://dx.doi.org/10.3389/fpls.2014.00105

Bao T., Sun T., Sun L. 2011. Effect of cadmium hyperaccumulation on antioxidative defense and proline accumulation of Solanum nigru L. African Journal of Biotechnology, 10: 7198-7206

Benavides M. P., Gallego S. M., Tomaro M. L. 2005. Cadmium toxicity in plants. Brazilian Journal of Plant Physiology, 17: $21-34$

http://dx.doi.org/10.1590/S1677-04202005000100003

Çanakci S., Karaboğa Z. 2013. Some physiological and bichemical responses to cadmium in salicylic acid applied cucumber (Cucumis sativus L.) seedlings. Pakistan Journal of Botany, 45: 1963-1968

Chakraborty S., Mukherjee A., Khuda-Bukhsh A. R., Das T. K. 2014. Cadmium-induced oxidative stress tolerance in cadmium resistant Aspergillus foetidus: its possible role in cadmium bioremediation. Ecotoxicology and Environmental Safetv. 106: 46-53 http://dx.doi.org/10.1016/j.ecoenv.2014.04.007

Ci D., Jiang D., Wollenweber B., Dai T., Jing Q., Cao W. 2010 Cadmium stress in wheat seedlings: growth, cadmium accumulation and photosynthesis. Acta Physiologiae Plantarum. 32: 365-373 http://dx.doi.org/10.1007/s11738-009-0414-0

Dong J., Wu F., Zhang G. 2006. Influence of cadmium on antioxidant capacity and four microelement concentrations in tomato seedlings (Lycopersicon esculentum). Chemosphere. 64: 1659-1666 http://dx.doi.org/10.1016/j.chemosphere.2006.01.030

Feng J., Shi Q., Wang X., Wei M., Yang F., Xu H. 2010. Silicon supplementation ameliorated the inhibition of photosynthesis andnitrate metabolism by cadmium $(\mathrm{Cd})$ toxicity in Cucumis sativus L. Scientia Horticulturae. 123: 521-530 http://dx.doi.org/10.1016/j.scienta.2009.10.013

Gao S., Yan R., Cao M., Yang W., Wang S., Chen F. 2008. Effects of copper on growth, antioxidant enzymes and phenylalanine ammonialyase activities in Jatropha curcas L. seedling. Plant Soil and Environment, 54: 117-122

Li Y., Zhang S. S., Jiang W. S., Liu D. H. 2013. Cadmium accumulation, activities of antioxidant enzymes, and malondialdehyde (MDA) content in Pistia stratiotes L. Environmental Science and Pollution Research, 20: 1117 1123 http://dx.doi.org/10.1007/s11356-012-1054-2 
LiZ.W., Huang J.A. 2014. Effects of nanoparticle hydroxyapatite on growth and antioxidant system in pakchoi (Brassica chinensis L.) from cadmium-contaminated soil. Journal of Nanomaterials, article ID 470962. http://dx.doi.org/10.1155/2014/470962

Lin L., Liao M., Mei L. 2012. A review of cucumber under cadmium stress. Asia Pacific Conference on Environmental Science and Technology (APEST 2012), 6: 443-446

Nagajyoti P. C., Lee K. D., Sreekanth T. V. M. 2010. Heavy metals, occurrence and toxicity for plants: a review. Environmental Chemistry Letters. 8: 199-216 http://dx.doi.org/10.1007/s10311-010-0297-8

Nazar R., Iqbal N., Masood A., Khan M. R., Syeed S., Khan N. A. 2012. Cadmium toxicity in plants and role of mineral nutrients in its alleviation. American Journal of Plant Sciences. 3: 1476-1489 http://dx.doi.org/10.4236/ajps.2012.310178

Pal'ove-Balang P., Kisová A., Pavlovkin J., Mistrík I. 2006. Effect of manganese on cadmium toxicity in maize seedlings. Plant, Soil and Environment, 52: 143-149

Peng K., Luo C., You W., Lian C.H., Li X., Shen Z. 2008. Manganese uptake and interactions with cadmium in the hyperaccumulator Phytolacca americana L. Journal of Hazardous Material, 154: 674-681 http://dx.doi.org/10.1016/j.jhazmat.2007.10.080

Scandalios J. G. 2005. Oxidative stress: molecular perception and transduction of signals triggering antioxidant gene defenses. Brazilian Journal of Medical and Biological Research. 38: 995-1014 http://dx.doi.org/10.1590/S0100-879X2005000700003

Shi G., Liu C., Cai Q., Liu Q., Hou C. 2010. Cadmium accumulation and tolerance of two safflower cultivars in relation to photosynthesis and antioxidantive enzymes. Bulletin of Environmental and Contamination Toxicology, 85: 256-263 http://dx.doi.org/10.1007/s00128-010-0067-0

Sperotto R. A., Ricachenevsky F. K., Williams L. E., Vasconcelos M. W., Menguer P. K. 2014. From soil to seed: micronutrient movement into and within the plant. Frontiers in Plant Science. 5: 438 http://dx.doi.org/10.3389/fpls.2014.00438

Tewari A., Singh R., Singh N. K., Rai U. N. 2008. Amelioration of municipal sludge by Pistia stratiotes L.: role of antioxidant enzymes in detoxification of metals. Bioresource Technology, 99: 8715-8721 http://dx.doi.org/10.1016/j.biortech.2008.04.018

Turner D. W., Fortescue J. A., Thomas D. S. 2007. Environmental physiology of the bananas (Musa spp.). Brazilian Journal of Plant Physiology. 19: 463-484 http://dx.doi.org/10.1590/S1677-04202007000400013

Wang M., Zou J. H., Duan X. C., Jiang W. S., Liu D. H. 2007. Cadmium accumulation and its effects on metal uptake in maize (Zea mavs L.). Bioresource Technology, 98: 82-88 http://dx.doi.org/10.1016/j.biortech.2005.11.028

Ying R. R., Qiu R. L., Tang Y. T., Hua P. J., Qiu H., Chen H. R., Shi T. H., Morel J. L. 2010. Cadmium tolerance of carbon assimilation enzymes and chloroplast in $\mathrm{Zn} / \mathrm{Cd}$ hyperaccumulator Picris divaricata. Journal of Plant Physiology, 167: 81-87 http://dx.doi.org/10.1016/j.jplph.2009.07.005

Zhang H. Y., Jiang Y. N., He Z. Y., Ma M. 2005. Cadmium accumulation and oxidative burst in garlic (Allium sativum). Journal of Plant Physiology. 162: 977-984 http://dx.doi.org/10.1016/j.jplph.2004.10.001

Zhang S. S., Zhang H. M., Qin R., Jiang W. S., Liu D. H. 2009. Cadmium induction of lipid peroxidation and effects on root tip cells and antioxidant enzyme activities in Vicia faba L. Ecotoxicology, 18: 814-823 http://dx.doi.org/10.1007/s10646-009-0324-3

Zou J., Xu P., Lu X., Jiang W. S., Liu D. H. 2008. Accumulation of cadmium in three sunflower (Helianthus annuus L.) cultivars. Pakistan Journal of Botany, 40 (2): 759-765

Zou J. H., Yu K. L., Zhang Z. G., Jiang W. S., Liu D. H. 2009. Antioxidant response system and chlorophyll fluorescence in chromium (VI)-treated Zea mays L. seedlings. Acta Biologica Cracoviensia, series Botanica, 51: 23-33

ISSN 1392-3196 / e-ISSN 2335-8947

Zemdirbyste-Agriculture, vol. 102, No. 2 (2015), p. 193-200

DOI 10.13080/z-a.2015.102.025

\title{
Kadmio įtaka mineralų kaupimuisi, antioksidacinei sistemai ir dujų mainams agurkuose
}

\author{
S. Sun ${ }^{1}$, M. Li ${ }^{2,3}$, J. Zuo ${ }^{2}$, W. Jiang ${ }^{2}$, D. Liu $^{2}$ \\ ${ }^{1}$ Tianjin Normal universitetas, Kinija \\ ${ }^{2}$ Tianjin Normal universiteto Tianjin pagrindinė gyvūnų ir augalų atsparumo laboratorija, Kinija \\ ${ }^{3}$ Renhuai vidurinè mokykla Nr. 4, Kinija
}

\section{Santrauka}

Hidroponiniu būdu augintuose agurkuose tirta ịvairių koncentracijų $(10,50,100$ ir $500 \mu \mathrm{M})$ kadmio $(\mathrm{Cd})$ įtaka mangano (Mn) bei geležies (Fe) kiekiui, lipidų peroksidacijai, antioksidacinių fermentų aktyvumui ir fotosintezei. Tyrimų rezultatai parodè, kad kadmis pirmiausia kaupiasi agurkų šaknyse. Didinant kadmio koncentraciją tirpale, jo kiekis šaknyse ir daiguose padidejo, o mangano kiekis esmingai $(P<0,005)$ sumažejo. Didinant kadmio koncentraciją tirpale ir poveikio laiką, geležies kiekis augaluose turèjo tendenciją didèti. Kadmio sukeltas oksidacinis stresas ir lipidų peroksidacija paskatino MDA (malondialdehido) koncentracijos didejiimą agurkuose. Palyginus su katalazès aktyvumu, kadmis labiau skatino superoksido dismutazès ir peroksidazès aktyvumą. Tai rodo, kad superoksido dismutazė ir peroksidazè yra svarbesnès siekiant pašalinti kadmio sukeltą oksidacinị stresą agurkuose. Palaisčius kadmiu, agurkuose sumažèjo fotosintezès bei garavimo intensyvumas, žiotelių laidumas ir tarpląstelinė $\mathrm{CO}_{2}$ koncentracija.

Reikšminiai žodžiai: antioksidaciniai fermentai, fotosintezès parametrai, kadmis, lipidų peroksidacija, paprastasis agurkas. 